\title{
Zinc-Blende GeC Stabilized on GaN (001): An Ab Initio Study
}

\author{
J. H. Camacho-García $\mathbb{D}^{1},{ }^{1}$ Ma L. Ruiz-Peralta $\mathbb{D}^{1},{ }^{1}$ G. Hernández-Cocoletzi $\mathbb{D}^{,}{ }^{2}$ \\ A. Bautista-Hernández $\mathbb{D}^{\mathbb{D}},{ }^{3}$ M. Salazar-Villanueva $\mathbb{D}^{\mathbb{B}},{ }^{3}$ A. Escobedo-Morales $\mathbb{D},{ }^{1}$ \\ E. Chigo-Anota $\mathbb{D}{ }^{1}$ and J. C. Moreno-Hernández $\mathbb{D}^{2}$ \\ ${ }^{1}$ Universidad Autónoma de Puebla, Facultad de Ingeniería Química, Puebla 72570, Mexico \\ ${ }^{2}$ Universidad Autónoma de Puebla, Instituto de Física, Apartado Postal J-48, Puebla 72570, Mexico \\ ${ }^{3}$ Universidad Autónoma de Puebla, Facultad de Ingeniería, Puebla 72570, Mexico
}

Correspondence should be addressed to J. H. Camacho-García; humberto.camacho@correo.buap.mx

Received 27 July 2021; Accepted 4 January 2022; Published 30 January 2022

Academic Editor: Sefer Bora Lisesivdin

Copyright (๑) 2022 J. H. Camacho-García et al. This is an open access article distributed under the Creative Commons Attribution License, which permits unrestricted use, distribution, and reproduction in any medium, provided the original work is properly cited.

\begin{abstract}
First-principle calculations have been performed to explore the initial stages of the zinc blende-like germanium carbide epitaxial growth on the gallium nitride $(001)-(2 \times 2)$ surface. First, we studied the $\mathrm{Ge} / \mathrm{C}$ monolayer adsorption and incorporation at high symmetry sites. Results show that the adsorptions at the top and hcp 1 sites are the most stable structures of $\mathrm{C}$ and $\mathrm{Ge}$, respectively. Different terminated surfaces were used on the $\mathrm{GeC}$ epitaxial growth. According to the surface formation energies, only the first two bilayers are stable; therefore, the $\mathrm{GeC}$ epitaxial growth is favorable only under N-rich conditions on a Ge-terminated surface and with Ge bilayers terminated. In addition, it is demonstrated that $\mathrm{GeC}$ bilayers on the C-terminated surfaces are unstable and preclude the epitaxial growth. Electronic properties have been investigated by calculating the density of states (DOS) and the projected density of states (PDOS) of the most favorable structures.
\end{abstract}

\section{Introduction}

Germanium carbide $(\mathrm{GeC})$ structures have attracted considerable interest because of the extensive applications in optoelectronic devices. Because they exhibit a narrow optical bandgap, they may be used in photovoltaic and infrared optical devices due to the low stress, tuned refractive index, and high absorption coefficient.

First-principle calculations based on the density functional theory (DFT) have been applied extensively due to the versatility to study different materials in order to calculate electronic, optical, and magnetic properties. In this regard, DFT calculations suggest that $\mathrm{Ge}_{\mathrm{x}} \mathrm{C}_{1-\mathrm{x}}$ thin films possess tunable bandgap [1-5]. In addition, amorphous films have been fabricated by radio frequency $(\mathrm{RF})$ reactive sputtering; the refractive index varied according to the synthesis conditions, an important feature in designing antireflection coatings [6]. Theoretical calculations have been developed to investigate the structural, electronic, optical, and magnetic properties of GeC. Majidi et al. reported DFT studies of the electronic and linear response optical properties of graphene-like XC (X=Si, Ge, and $\mathrm{Sn})$ nanosheets, and the results showed a bandgap of $2.1 \mathrm{eV}$ [7]. Xu et al. investigated magnetic behavior of $\mathrm{B}, \mathrm{N}$, and $3 \mathrm{~d}$ transition metal substitutions in germanium carbide monolayers. Results indicated that impurity atoms change the magnetic moment. In the 2D-GeC system, in both substituted Ge sublattice(VGe@ $\mathrm{Ni})$ or C sublattice (VC@Ni), the distributions of magnetic moments are centered on $\mathrm{Ni}$ and the nearest $\mathrm{Ge} / \mathrm{C}$ atoms [8]. The obtained magnetic nanostructures are technologically significant for applications in spintronics devices. There are several theoretical investigations of $\mathrm{GeC}$ in the zinc blende $(\mathrm{zb})$ phase in which the reported lattice parameter values are $4.43 \AA$ - $-4.62 \AA$ [ [9-13]. The calculated band structure shows an indirect bandgap with values from $1.52 \mathrm{eV}$ to $2.56 \mathrm{eV}$ [14-17]. Zhang et al. [12] used the plane-wave pseudopotential (PP) approach within the density functional perturbation theory to investigate the lattice dynamics and 
thermodynamic properties of $\mathrm{SiC}, \mathrm{GeC}$, and $\mathrm{SnC}$. The obtained phonon dispersion exhibits no imaginary phonon frequencies of $\mathrm{zb}-\mathrm{GeC}$, indicating the structural stability. Furthermore, structural parameters and electronic and chemical bonding properties of $\mathrm{zb}-\mathrm{GeC}$ (100) surfaces were investigated through DFT, and results revealed that Geterminated surfaces exhibit metallic properties [18]. However, the experimental confirmations are not available yet.

Wide-bandgap semiconductors that have bandgaps greater than $3.0 \mathrm{eV}$, such as $\mathrm{SiC}, \mathrm{ZnO}, \beta-\mathrm{Ga}_{2} \mathrm{O}_{3}$, and $\mathrm{GaN}$ [19], have received attention due to their physical properties and different applications [20]. In this regard, gallium nitride $(\mathrm{GaN})$ has been applied in light-emitting diodes [21], X-ray detectors [22], and manometer devices [23]. GaN crystallizes in the wurtzite $(w)$ and zinc blende phases with $a_{0}=3.188 \AA$, $c=5.185 \AA$, and $a_{0}=4.52 \AA$, respectively [24]. There are experimental reports confirming the existence of stable GaN in the zinc blende phase [25-28]. Growth of this material is obtained by means of thin films, on $\beta$-SiC and GaAs substrates, in the (001) direction, in a temperature range from $700^{\circ} \mathrm{C}$ to $200^{\circ} \mathrm{C}[25,26]$. However, Pak et al. [27] and Novikov et al. [28] demonstrated the stability at room temperature of the $\mathrm{zb}-\mathrm{GaN}$ (001) films grown by the molecular beam epitaxy (MBE) technique on (001) $\mathrm{MgO}$ and (001) GaAs, respectively; e.g., Figure 6 in [28], shows a $100 \mu \mathrm{m}$-thick zinc-blende GaN layer grown on a 3 -inch GaAs substrate. Results show outstanding photoluminescence (PL) emission [29], which suggests that this is a suitable material for optical applications. In addition, zbGaN structure is isotropic, which avoids lattice mismatch between $\mathrm{GaN}$ and InGaN barriers in order to fabricate lightemitting diodes with higher efficiency. GaN-based UV photodetectors showed a significantly enhanced photoresponsivity near the $365 \mathrm{~nm}$ wavelength range (UV-A region) [30].

In the case of $\mathrm{GeC}$, reports show that thin film growth results in amorphous GeC. For this reason, this work explored the possibility of using $\mathrm{GaN}$ as a substrate to obtain an epitaxial growth of $\mathrm{zb}-\mathrm{GeC}$.

The development of electronics based on gallium nitride substrates has been focused on structural defect elimination to achieve better electrical and thermal conductivities [31]. In this sense, Posadas et al. reported the fabrication of $\mathrm{YMnO}_{3}$ epitaxial thin films on $\mathrm{w}-\mathrm{GaN} /$ sapphire and determined the most stable configuration from calculations of the formation energy of the interface [32]. $\mathrm{Al}_{\mathrm{x}} \mathrm{Ga}_{1-\mathrm{x}} \mathrm{N}$ ultraviolet avalanche photodiodes were grown on GaN substrates; results show that the avalanche gain is due to the low dislocation density in the bulk of GaN substrates [33]. Moreover, heterostructures of $\mathrm{GaN}$ with IV compounds have been studied, either theoretically or experimentally, and they are found to be stable [34-37]. Recently, electronic structure, charge transfer, and optical properties of GaN$\mathrm{GeC}$ van der Waals heterostructures have been investigated using first-principle calculations; results suggest optical absorption in the visible region, which indicates that this is a promising material for photocatalytic applications [38]. In addition, tunable photocatalysis properties of $\mathrm{GaN} / \mathrm{GeC}$ have been used for the production of $\mathrm{H}_{2}$ fuel [39], water splitting, and solar energy conversion. The development of new materials involves property investigation in order to reach a real application. $\mathrm{GaN} / \mathrm{GeC}$ is a promising material, and since $\mathrm{zb}-\mathrm{GeC}$ and $\mathrm{GaN}$ are materials with potential applications in different fields, it is important to study the epitaxial growth to achieve functional devices. In this regard, both compounds have the same crystal structure, and their lattice parameter mismatch is smaller than $15 \%$; it is possible to grow $\mathrm{GeC} / \mathrm{GaN}$ systems. In this work, $\mathrm{Ge}$ and $\mathrm{C}$ monolayer/bilayer formation on the $\mathrm{GaN}$ (001) surface was studied by first-principle calculations in order to investigate the initial stages of the $\mathrm{zb}-\mathrm{GeC}$ formation. In addition, the formation energies were calculated to demonstrate the stability of the studied configurations. The density of states (DOS) and structural, thermodynamical, and electronic properties were also calculated. This paper is organized as follows: in Section 2, we briefly describe the computational methodology, results are discussed in Section 3, and finally, conclusions are presented.

\section{Computational Methodology}

Zinc-blende gallium nitride (001) surface is considered a substrate of germanium carbide $(\mathrm{GeC})$. Total energy calculations have been carried out using the periodic density functional theory as implemented in the PWscf code of the Quantum ESPRESSO package [40]. Exchange-correlation interactions are approximated with the generalized gradient approximation (GGA), as stated in the Perdew-Burke-Ernzerhof (PBE) parametrization [41]. The electron-ion interactions are treated according to the pseudopotential approach, employing Vanderbilt ultrasoft pseudopotentials [42]. Electronic states are expanded in plane waves with the cutoff energy of $30 \mathrm{Ry}$ and charge density of $240 \mathrm{Ry}$. For convergence in geometry optimization, all force components should be smaller than $0.026 \mathrm{eV} /$ $\AA$, and the total energy differences must be less than $0.0026 \mathrm{eV}$. A $7 \times 7 \times 1$ Monkhorst-Pack mesh [43] has been used to sample the Brillouin zone. The surface is modeled using the supercell method. The supercell is formed by a slab (composed of $4 \mathrm{GaN}$ bilayers) and a vacuum gap width greater than $15 \AA$. Fractional pseudo-hydrogen atoms saturate the bottom surface dangling bonds in order to eliminate the possibility of undesirable charge transfer. To determine the density of states (DOS), the $k$-point mesh is increased to $21 \times 21 \times 1$.

\section{Results and Discussion}

3.1. Adsorption and Incorporation of the Ge and C Monolayer. Figure 1 describes the non-reconstructed GaN (001)- $(2 \times 2)$ surface and the high symmetry sites in the current study. For (001) zb-like surfaces, the high symmetry sites are defined as top, hcp, fcc, and hcpl [44]. The top site is obtained when a $\mathrm{Ge} / \mathrm{C}$ monolayer (ML) is placed on top of the first Ga-ML. At the hcp site, the Ge/C atoms are adsorbed on top of the first $\mathrm{N}$-ML. The fcc site is obtained when the adatoms are adsorbed over $\mathrm{Ga}$ atoms from the second monolayer, and the hcp 1 geometry is formed when $\mathrm{Ge} / \mathrm{C}$ atoms are adsorbed 


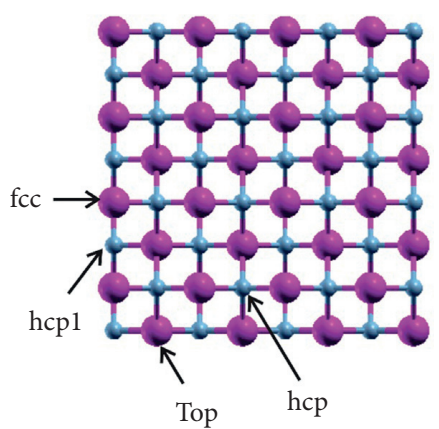

(a)

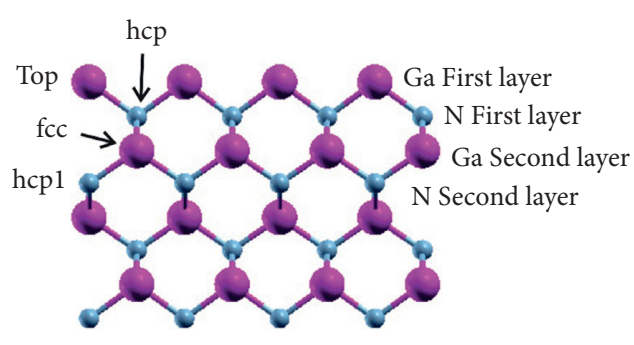

(b)

Figure 1: Schematic atomic structure for the Ga-terminated GaN $(001)-(2 \times 2)$ surface, showing high symmetry sites (top, hcp, fcc, and hcp1) for the monolayer Ge/C adsorption. (a) The top view. (b) The side view.

above the second $\mathrm{N}$ monolayer (Figure 1). In addition, we explored the $\mathrm{Ge} / \mathrm{C}$ monolayer incorporation into the $\mathrm{GaN}$ (001)- $(2 \times 2)$ surface, where $\mathrm{Ge}$ or $\mathrm{C}$ atoms displace the $\mathrm{Ga}$ atoms, yielding $\mathrm{Ga}$ as the new adatoms that are adsorbed on the surface at four high symmetry sites. In order to study adsorption at high symmetry sites, layers (either $\mathrm{C}$ or $\mathrm{Ge}$ ) are placed on top of the GaN substrate; then, geometry is optimized to calculate the system total energy. In these calculations, the number of atoms of the system remains constant. Whence, a comparison can be made between the total energies of each configuration. Differences among calculated energies are referred to as relative energies. The relative energy analysis is shown in Table 1. This phenomenon has been previously studied for different surfaces [44-47] since this configuration stimulates the bilayer growth in some cases.

According to the results, the Ge monolayer adsorption at the hcp 1 site is the most stable structure, shown in Figure 2(a), whereas the Ge-ML adsorption at the hcp site is energetically unfavorable. However, the C-ML adsorption at the top site geometry is more stable than hcp, fcc, and hcp 1 sites by $1.07 \mathrm{eV} / \mathrm{C}$-atom, $1.02 \mathrm{eV} / \mathrm{C}$-atom, and $0.89 \mathrm{eV} / \mathrm{C}$-atom, respectively. As it is shown in Figure 2(b), the $\mathrm{C}$ dangling bonds at the top surface are saturated, forming $\mathrm{C}$ dimers with a bond length of $1.42 \AA$. In the Ge incorporation, the hcp 1 site is more stable than the top, fcc, and hcp sites by $0.048 \mathrm{eV} / \mathrm{Ge}-$ atom, $0.058 \mathrm{eV} / \mathrm{Ge}$-atom, and $0.066 \mathrm{eV} / \mathrm{Ge}$-atom, respectively. The hcp site exhibits as favorable geometry, followed by the top, hcpl, and fcc (hcp >top $>h c p 1>f c c)$ for the $C$ incorporation into the $\mathrm{GaN}$ surface.

In addition, we have studied different $\mathrm{Ge} / \mathrm{C}$-terminated $\mathrm{GaN}(001)$ surfaces, which indicates that the structure is not gallium terminated; instead, the $\mathrm{Ge} / \mathrm{C}$ monolayer may be grown. Figure 3 shows the optimized structures, and the structural stabilities will be discussed by means of the formation energy analysis.

3.2. GeC Formation. In order to investigate the epitaxial growth of $\mathrm{GeC}$ on the $\mathrm{GaN}$ (001) surface, we have used the structures of $\mathrm{Ge} / \mathrm{C} \mathrm{ML}$ adsorption and different $\mathrm{Ge} / \mathrm{C}$-terminated GaN (001) surfaces; the most stable geometries are displayed in Figure 4. Figure 4(a) shows the epitaxial growth of the $\mathrm{GeC}$ bilayer on the Ge-terminated $\mathrm{GaN}$ (001) ideal surface. Adding a $\mathrm{C}$ monolayer to the $\mathrm{Ge}$ adsorption at the hcp1 site formed this structure. Figure 4(b) exhibits the C dimer formation, with a bond length of $1.47 \AA$, when a C monolayer is deposited on the Ge-terminated GaN surface, demonstrating that the surface reconstruction avoids the epitaxial growth because of the carbon broken bonds' saturation [48]. Figure 4(c) corresponds to the formation of a $\mathrm{GeC}-\mathrm{ML}$ on the Ge-terminated GaN surface, which is obtained when a Ge ML is added on the $\mathrm{C}$ dimers (Figure 4(b)). In the top view of the figure, atoms of $\mathrm{Ge}$ and $\mathrm{C}$ show a "chair" distorted configuration [49] as the Ge-Ge bond lengths $(2.76 \AA)$ are larger than the $\mathrm{C}-\mathrm{C}$ bond lengths $(1.35 \AA)$ with the bond angles of $106.1^{\circ}$.

Figure 5 shows the epitaxial growth of $\mathrm{GeC}$ on the Geterminated $\mathrm{GaN}$ surface. Figure 5(a) depicts a $\mathrm{GeC}$ bilayer, while Figure 5(b) displays the atomic structure of the double $\mathrm{GeC}$ bilayer formation, which as Che et al. [18] reported. Therefore, the $\mathrm{GeC}$ growth is more favorable on Ge-terminated surface. The bond length between Ge-C is $1.95 \AA$, which agrees well with previous theoretical reports [50]. Since we have surfaces with a different number of atoms to determine the most stable structure, the surface formation energy (SFE) formalism is employed as described below.

3.3. Surface Formation Energy. To determine the stability of all models, we have used the surface formation energy (SFE) formalism [45-51]. In order to apply the SFE formalism, we consider thermodynamic equilibrium between the surface and the bulk. This implies that

$$
\mu_{\mathrm{GaN}(\mathrm{bulk})}=\mu_{\mathrm{Ga}}+\mu_{N}-\Delta H_{f}^{\mathrm{GaN}},
$$

with

$$
\begin{aligned}
& \mu_{\mathrm{Ga}} \leq \mu_{\mathrm{Ga}}^{\text {bulk }}, \\
& \mu_{N} \leq \mu_{N_{2}}^{\text {molecule }},
\end{aligned}
$$

where $\mu_{i}$ is the chemical potential of the $i^{\text {th }}$ species and $\Delta H_{f}^{\mathrm{GaN}}$ is the enthalpy or formation heat of $\mathrm{GaN}$ in the bulk structure. According to calculations, $\Delta H_{f}^{\mathrm{GaN}}=0.9 \mathrm{eV}$, in agreement with previous reports [45]. Provided that 
TABLE 1: Relative adsorption energies $(\mathrm{eV})$ and the incorporation of different Ge and C coverage.

\begin{tabular}{lccc}
\hline Site & Relative energy & Site & Relative energy \\
\hline Ge-top & 0.069 & C-top & $\mathbf{0 . 0 0 0}$ \\
Ge-hcp & 0.148 & C-hcp & 1.073 \\
Ge-fcc & 0.104 & C-fcc & 1.022 \\
Ge-hcp1 & $\mathbf{0 . 0 0 0}$ C-hcp1 & 0.892 \\
Ge- $^{\text {st }}$ layer/Ga-top & 0.118 & C- $1^{\text {st }}$ layer/Ga-top & 0.285 \\
Ge- $^{\text {st }}$ layer/Ga-hcp & 0.136 & $\mathrm{C}-1^{\text {st }}$ layer/Ga-hcp & 0.078 \\
Ge-1 $^{\text {st }}$ layer/Ga-fcc & 0.128 & $\mathrm{C}-1^{\text {st }}$ layer/Ga-fcc & 0.987 \\
Ge- $^{\text {st }}$ layer/Ga-hcp1 & 0.070 & $\mathrm{C}-1^{\text {st layer/Ga-hcp1 }}$ & 0.445 \\
\hline
\end{tabular}

Zero energy (bold numbers) is the energy reference in each case.
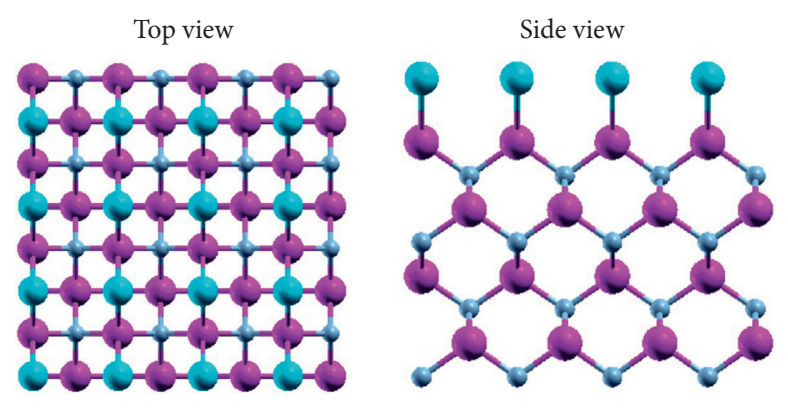

(a)

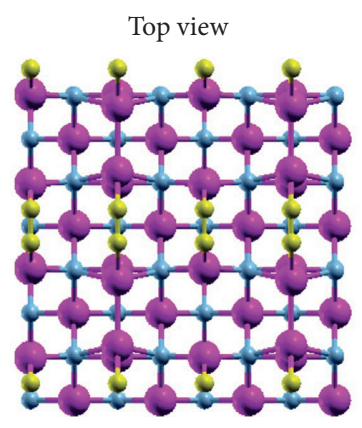

(b)

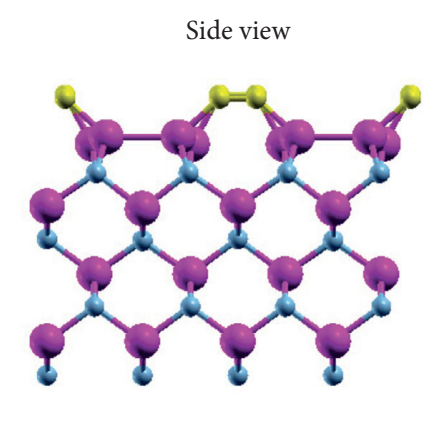

(b)

Figure 2: (a) Ge adsorption at the hcp1 site and (b) C adsorption at the top site.

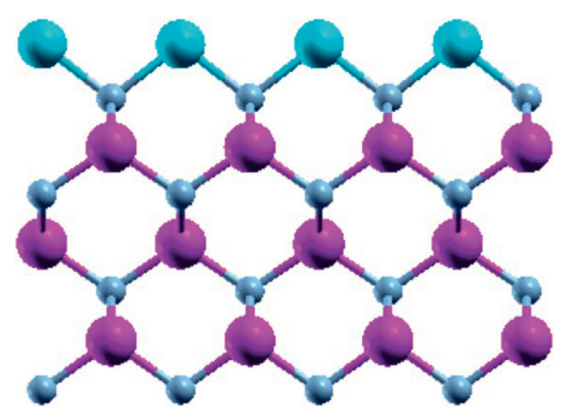

(a)

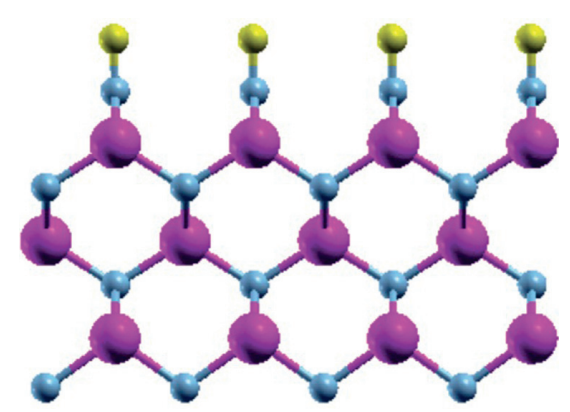

(b)

Figure 3: Optimized structures for (a) Ge- and (b) C-terminated surfaces.

constant pressure and zero temperature are assumed, the SFE may take the following form:

$$
E_{f}=E_{\text {slab }}-E_{\text {ref }}-\Delta n_{\mathrm{Ga}} \mu_{\mathrm{Ga}}-\Delta n_{N} \mu_{N}-\Delta n_{\mathrm{Ge}} \mu_{\mathrm{Ge}}-\Delta n_{\mathrm{C}} \mu_{\mathrm{C}},
$$

where $E_{\text {slab }}$ is the energy of each model and $E_{\text {ref }}$ is the reference energy. In this case, the reference is the ideally Gaterminated GaN $(001)-(2 \times 2)$ surface. $\Delta n_{i}$ is the excess or deficit of the $i^{\text {th }}$ atom. The SFE is a function of the Ga chemical potential with lower limit (N-rich conditions) $\mu_{\mathrm{Ga}}=\mu_{\mathrm{Ga}}^{\mathrm{bulk}}-\Delta H_{f}^{\mathrm{GaN}}$ and upper limit (Ga-rich conditions) $\mu_{\mathrm{Ga}}=\mu_{\mathrm{Ga}}^{\mathrm{balk}} \cdot \mu_{\mathrm{Ge}}$ and $\mu_{\mathrm{C}}$ are obtained employing a diamond structure. The obtained results are shown in Figure 6; under Ga-rich conditions, the adsorption of Ge at the hcp1 site is the most stable configuration, whereas the C-terminated surface is the most unfavorable. On the contrary, the Geterminated surface is the most stable structure. The $\mathrm{C}$ adsorption structures (Figures 2(b) and 4(b)) have a gain in energy due to the rearrangement of the $\mathrm{C}$ atoms to form dimers, which have a bond length of $1.24 \AA$. However, both structures are unstable. On the contrary, Figure 6(b) shows that, under N-rich conditions, the epitaxial growth of 1 or 2 $\mathrm{GeC}$-monolayers on the Ge-terminated surface is possible. However, as the GeC-ML number rises, the system becomes unstable due to an increasing SFE, which could be explained by several studies of $\mathrm{GeC}$ thin films [3, 4, 6, 49-54].

3.4. Electronic Properties. In this section, the total density of states (DOS) and the projected density of states (PDOS) of the most stable structures are calculated to show the electronic contributions to the Fermi level.

The DOS and PDOS are plotted as functions of energy, with the Fermi level being the zero energy. Notice that we outlined the contributions to the p-orbitals of each element. 

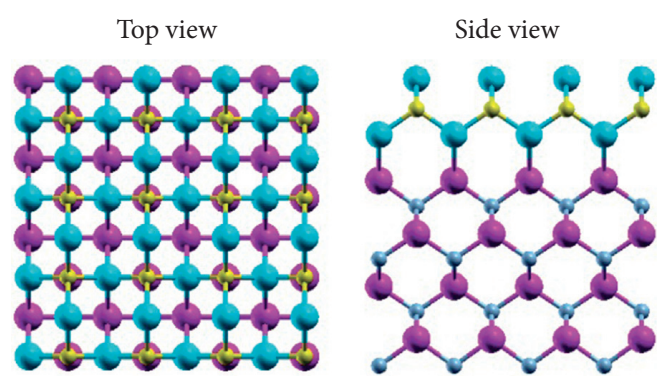

(a)

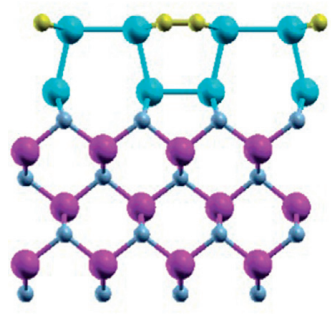

Top view

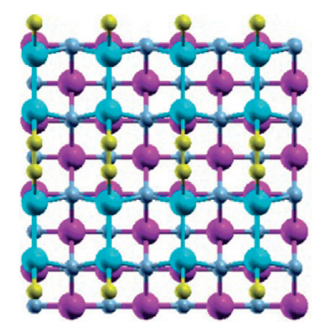

(b)
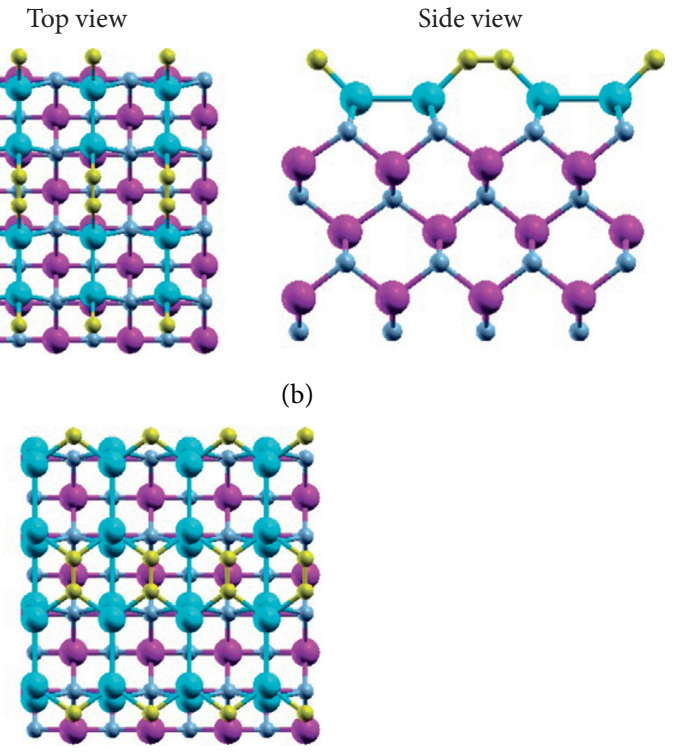

(c)

Figure 4: Different GeC surfaces: (a) formation of a GeC bilayer on the ideal Ga-terminated surface (GaN/GeC), (b) the C dimers' formation on the Ge-terminated surface (Ge-term-C), and (c) GeC chair-like layer.

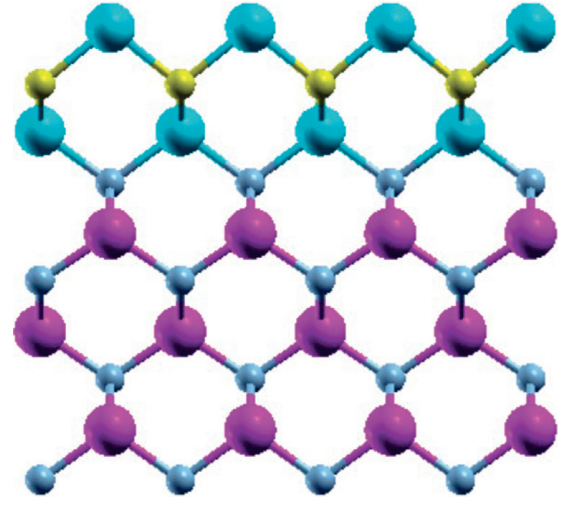

(a)

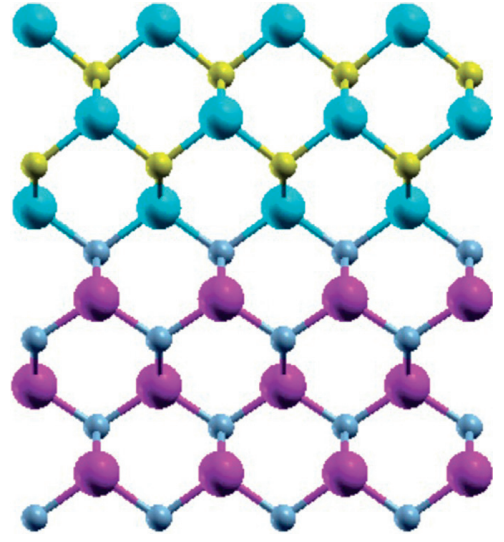

(b)

Figure 5: Epitaxial growth of (a) a GeC bilayer and (b) two $\mathrm{GeC}$ bilayers on the Ge-terminated surface.

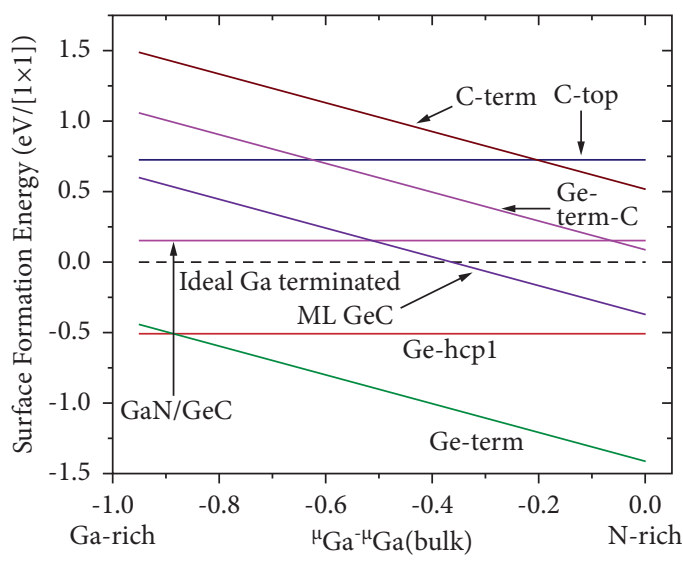

(a)

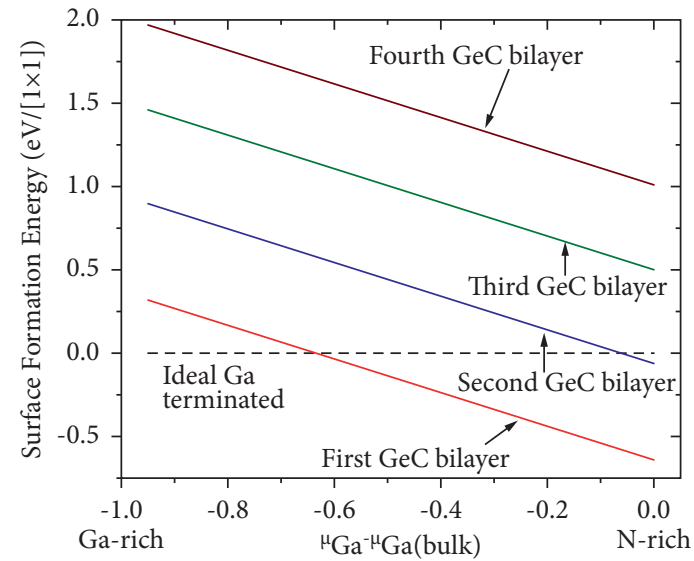

(b)

Figure 6: Surface formation energy (SFE) as a function of the Ga chemical potential for (a) different structures and (b) epitaxial growth. 


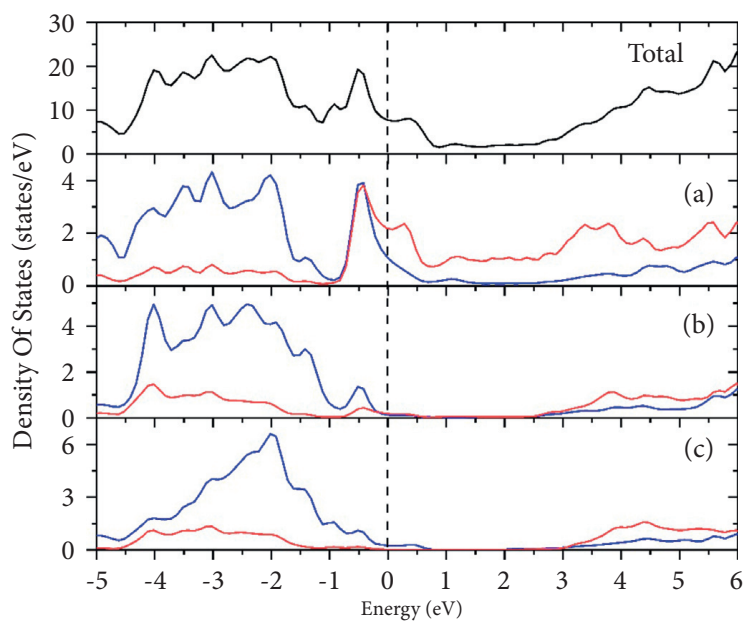

Figure 7: DOS and PDOS for the GaN (001) surface: (a), (b), and (c) exhibit the PDOS of the $1^{\text {st }}, 2^{\text {nd }}$, and $3^{\text {rd }}$ GaN bilayers, respectively. The red and blue lines correspond to Ga-p and N-p, respectively.

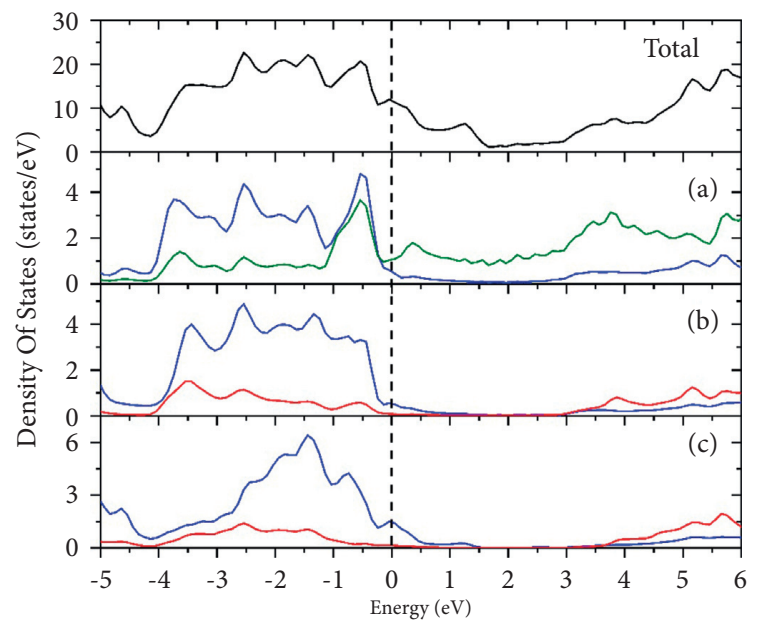

FIGURE 8: DOS and PDOS for the Ge-terminated surface: (a), (b), and (c) exhibit the PDOS for the $1^{\text {st }}, 2^{\text {nd }}$, and $3^{\text {rd }} \mathrm{GaN}$ bilayers, respectively. The red lines correspond to Ga-p, the blue lines are the contributions of $\mathrm{N}-\mathrm{p}$, and Ge-p is represented as olive lines.

Figure 7 shows the electronic properties of the GaN (001)$(2 \times 2)$ clean surface; the second and third GaN bilayers show a bandgap of $\approx 2 \mathrm{eV}$, according to previous reports [55]. Below the Fermi level, the major contributions are given by the N-p orbitals. On the contrary, the main contribution above the Fermi level is obtained from Ga-p orbitals. However, the first GaN bilayer shows a metallic behavior due to the Ga-terminated surface.

Figure 8 exhibits the DOS and PDOS of the Ge-terminated surface; the last two bilayers remain as semiconductors with a bandgap of $2 \mathrm{eV}$. Below the Fermi level, $\mathrm{N}$-p orbitals make the main contribution to the DOS. In the first bilayer, the change of $\mathrm{Ga}$ atoms by $\mathrm{Ge}$ atoms does not affect the surface metallic behavior, and also, there is no dimerization due to the electronic reconstruction. Just below the Fermi level, the main contribution to the DOS is due to the N-p orbitals. An additional GeC bilayer (Figure 9) modifies the contributions on the first bilayer. Close to the Fermi level (in -1 to $-2 \mathrm{eV}$ range), Ge-p orbitals are the major contributors

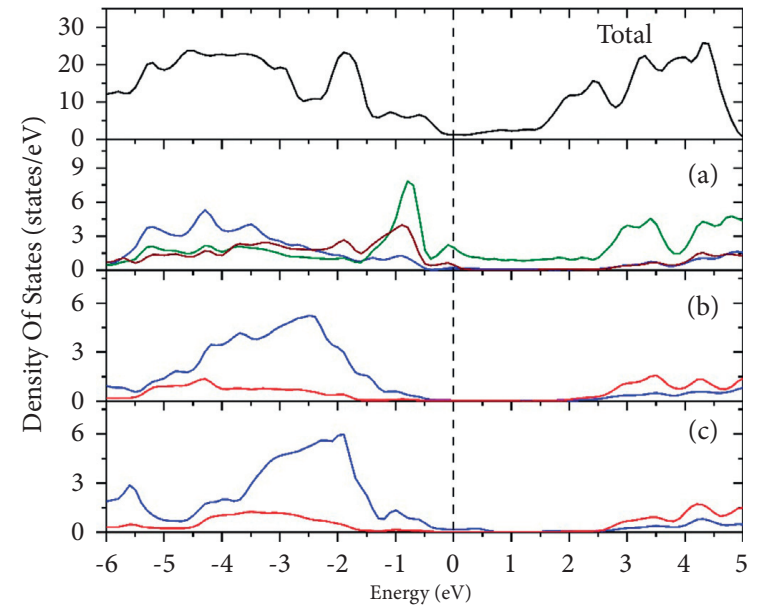

FIgURE 9: DOS and PDOS for a bilayer GeC. The contribution of Ga-p (red lines), N-p (blue lines), Ge-p (olive lines), and C-p (wine lines) is illustrated.

to the DOS, no change above the Fermi level is manifested, and the surface unoccupied states are the Ge-p orbitals.

\section{Conclusions}

We have performed ab initio calculations to investigate the initial stages of the $\mathrm{GeC}$ structure formation on the $\mathrm{GaN}$ (001)- $(2 \times 2)$ surface. The adsorption of a monolayer of $\mathrm{C}$ and Ge is studied. Results show that the most stable configurations correspond to the $\mathrm{C}$ and $\mathrm{Ge}$ adsorption at the top and hcp1 sites, respectively. The epitaxial growth of GeC bilayers should be obtained with a control technique of deposition since the calculated SFE shows that only the first two bilayers are stable only under N-rich conditions and on a Ge-terminated surface. Subsequently, the GeC surface becomes unstable due to the relationship between the number of layers and the SFE, in agreement with experimental reports. In addition, our results confirmed those reported by Che et al. [18], which indicate that the Geterminated slab surface is more stable than the C-terminated 
surface. On the contrary, the GeC bilayer on the C-terminated surface is unstable and, moreover, avoids the epitaxial growth provided that the carbon broken bonds saturate. The DOS graphs indicate that all the stable surfaces have a metallic behavior as no energy gap is manifested at the Fermi level.

\section{Data Availability}

The datasets generated and/or analysed during the current study are available from the corresponding author upon reasonable request. Please contact humberto.camacho@ correo.buap.mx

\section{Conflicts of Interest}

The authors declare that they have no conflicts of interest.

\section{Acknowledgments}

The authors thankfully acknowledge the computer resources, technical advice, and support provided by Laboratorio Nacional de Supercómputo del Sureste de México (LNS), a member of the CONACYT network of national laboratories. They would like to acknowledge the computer resources and support provided by Instituto de Física, BUAP.

\section{References}

[1] K. Makoto, "Deposition of new microcrystalline materials, $\mu \mathrm{c}-\mathrm{SiC}, \mu \mathrm{c}-\mathrm{GeC}$ by HWCVD and solar cell applications," Thin Solid Films, vol. 516, no. 5, Article ID 490, 2008.

[2] L. B. Drissi and F. Z. Ramadan, "Excitonic effects in GeC hybrid: many-body Green's function calculations," Physica E: Low-dimensional Systems and Nanostructures, vol. 74, Article ID 377, 2015.

[3] X. S. Che, Z. T. Liu, Y. P. Li, and N. Wang, "Effects of hydrogen and substrate temperature on the chemical bonding and optical properties of germanium carbon films deposited by reactive sputtering," Applied Surface Science, vol. 258, Article ID 6212, 2012.

[4] C. Q. Hu, J. Q. Zhu, W. T. Zheng, and J. C. Han, “Annealing effects on the bonding structures, optical and mechanical properties for radio frequency reactive sputtered germanium carbide films," Applied Surface Science, vol. 255, Article ID 3552, 2009.

[5] Z. Xu, Y. Li, C. Li, and Z. Liu, "Tunable electronic and optical behaviors of two-dimensional germanium carbide," Applied Surface Science, vol. 367, Article ID 19, 2016.

[6] Z. Liu, J. Zhu, N. K. Xu, and X. L. Zheng, "Structure and properties of germanium carbide films prepared by RF reactive sputtering in Ar/ $\mathrm{CH}_{4}$," Japanese Journal of Applied Physics, vol. 36, Article ID 3625, 1997.

[7] S. Majidi, S. M. Elahi, A. Esmailian, and F. Kanjouri, "First principle study of electronic and optical properties of planar $\mathrm{GeC}, \mathrm{SnC}$ and $\mathrm{SiC}$ nanosheets," Protection of Metals and Physical Chemistry of Surfaces, vol. 53, Article ID 773, 2017.

[8] Z. Xu, Y. Li, and Z. Liu, "Electronic and magnetic behaviors of $\mathrm{B}, \mathrm{N}$, and $3 \mathrm{~d}$ transition metal substitutions in germanium carbide monolayer," Journal of Magnetism and Magnetic Materials, vol. 451, Article ID 799, 2018.
[9] W. Sekkal and A. Zaoui, "Predictive study of thermodynamic properties of GeC," New Journal of Physics, vol. 4, p. 1, 2002.

[10] A. F. Goncharov, E. V. Yakovenko, and S. M. Stishov, "Stability of silicon carbide at high pressures," Pis'ma $v$ Zhurnal Èksperimental'noi i Teoreticheskoi Fiziki, vol. 52, Article ID 1092, 1990.

[11] H. Rücker and M. Methfessel, "Anharmonic Keating model for group-IV semiconductors with application to the lattice dynamics in alloys of Si, Ge, and C," Physical Review B, vol. 52, pp. 11059-11061, 1995.

[12] X. Zhang, S. Quan, C. Ying, and Z. Li, “Theoretical investigations on the structural, lattice dynamical and thermodynamic properties of $\mathrm{XC}(\mathrm{X}=\mathrm{Si}, \mathrm{Ge}, \mathrm{Sn})$," Solid State Communications, vol. 151, Article ID 1545, 2011.

[13] W. Sukkabot, "Transition-metal control of electronic and magnetic properties in $\mathrm{GeC}$ semiconductor: spin density functional calculations," Physica Scripta, vol. 95, no. 3, pp. 035804-35811, 2020.

[14] R. Khenata, H. Baltache, M. Sahnoun, M. Driz, M. Rétat, and B. Abbar, "Full potential Linearized Augmented Plane wave calculations of structural and electronic properties of $\mathrm{GeC}$, SnC and GeSn," Physica B: Condensed Matter, vol. 336, Article ID 321, 2003.

[15] A. Benzair and H. Aourag, "Energetic and electronic structure of the hypothetical cubic zincblende-like semiconductors GeC and SnC," Physica Status Solidi, vol. 231, Article ID 411, 2002.

[16] Q. J. Liu, Z. T. Liu, X. S. Che, L. P. Feng, and H. Tian, "Firstprinciples calculations of the structural, elastic, electronic, chemical bonding and optical properties of zinc-blende and rocksalt GeC," Solid State Science, vol. 13, Article ID 2177, 2011.

[17] R. J. Pandey, "A theoretical study of stability, electronic, and optical properties of $\mathrm{GeC}$ and SnC," Journal of Applied Physics, vol. 88, Article ID 6462, 2000.

[18] X. S. Che, Z. T. Liu, Y. P. Li, and Q. J. Liu, "First-principles calculations of zinc-blende $\mathrm{GeC}\left(\begin{array}{lll}0 & 0 & 1\end{array}\right)$ surfaces," Computational Materials Science, vol. 6, Article ID 121, 2013.

[19] M. Kim, J. H. Seo, U. Singisetti, and Z. Ma, "Recent advances in free-standing single crystalline wide band-gap semiconductors and their applications: $\mathrm{GaN}, \mathrm{SiC}, \mathrm{ZnO}, \beta-\mathrm{Ga}_{2} \mathrm{O}_{3}$, and diamond," Journal of Materials Chemistry $C$, vol. 5, Article ID 8338, 2017.

[20] F. Roccaforte, P. Fiorenza, G. Greco et al., "Emerging trends in wide band gap semiconductors ( $\mathrm{SiC}$ and $\mathrm{GaN}$ ) technology for power devices," Microelectronic Engineering, vol. 187-188, pp. 66-77, 2018.

[21] L. Y. Lee, "Cubic zincblende gallium nitride for greenwavelength light-emitting diodes," Materials Science and Technology, vol. 33, no. 5, p. 1, 2017.

[22] T. Gohil, J. Whale, G. Lioliou et al., "X-ray detection with zinc-blende (cubic) GaN Schottky diodes," Nature Scientific Reports, vol. 6, Article ID 29535, 2016.

[23] N. I. Kim, Y. L. Chang, J. Chen et al., "Piezoelectric pressure sensor based on flexible gallium nitride thin film for harshenvironment and high-temperature applications," Sensors Actuat A-Physics, vol. 305, Article ID 111940, 2020.

[24] A. Trampert, O. Brandt, and K. H. Ploog, "Chapter 7 crystal structure of group III nitrides," Semiconductors and Semimetals, vol. 50, pp. 167-192, 1997.

[25] A. D. Bykhovski and M. S. Shur, "Surface reconstruction of zinc-blende GaN," Applied Physics Letters, vol. 69, no. 16, pp. 2397-2399, 1996. 
[26] J. H. Kim and P. H. Holloway, "Wurtzite to zinc-blende phase transition in gallium nitride thin films," Applied Physics Letters, vol. 84, Article ID 711, 2004.

[27] J. Pak, W. Lin, K. Wang et al., "Growth of epitaxial iron nitride ultrathin film on zinc-blende gallium nitride," Journal of Vacuum Science \& Technology A, vol. 28, p. 4, 2010.

[28] S. V. Novikov, A. J. Kent, and C. T. Foxon, "Molecular beam epitaxy as a growth technique for achieving free-standing zinc-blende $\mathrm{GaN}$ and wurtzite $\mathrm{Al}_{\mathrm{x}} \mathrm{Ga}_{1-\mathrm{x}} \mathrm{N}$," Progress in Crystal Growth and Characterization of Materials, vol. 63, no. 2, pp. 25-39, 2017.

[29] M. Kumar, M. K. Rajpalke, B. Roul et al., "Temperaturedependent photoluminescence of $\mathrm{GaN}$ grown on $\beta-\mathrm{Si}_{3} \mathrm{~N}_{4} / \mathrm{Si}$ (111) by plasma-assisted MBE," Journal of Luminescence, vol. 131, Article ID 614, 2011.

[30] C. J. Lee, C. H. Won, J. H. Lee, H. Sung-Ho, and H. Park, "Selectively enhanced UV-A photoresponsivity of a $\mathrm{GaN}$ MSM UV photodetector with a step-graded AlxGa1-xN buffer layer," Sens, vol. 17, no. 8, pp. 1684-1691, 2017.

[31] T. Paskova, D. A. Hanser, and K. Evans, "GaN substrates for III-nitride devices," Proceedings of the IEEE, vol. 98, no. 7, Article ID 1324, 2010.

[32] A. Posadas, J. B. Yau, C. H. Ahn et al., "Epitaxial growth of multiferroic $\mathrm{YMnO}_{3}$ on GaN," Applied Physics Letters, vol. 87, Article ID 171915, 2005.

[33] D. Yoo, J. Limb, J. H. Ryou et al., "AlxGa1-xN ultraviolet avalanche photodiodes grown on GaN substrates," IEEE Photonics Technology Letters, vol. 19, no. 17, Article ID 1313, 2007.

[34] A. Rizzi, R. Lantier, and H. Lüth, "Boundary conditions and the macroscopic field at $\mathrm{SiC} / \mathrm{AlN}$ and $\mathrm{SiC} / \mathrm{GaN}$ heterostructures," Physica Status Solidi (A), vol. 177, Article ID 165, 2000.

[35] M. Sznajder, E. Wachowicz, and J. A. Majewski, “Ab initio studies of early stages of nitride growth process on silicon carbide," Journal of Crystal Growth, vol. 401, Article ID 25, 2014.

[36] J. Komiyama, Y. Abe, S. Suzuki, S. H. Nakanishi, and A. Koukitu, "MOVPE of AlN-free hexagonal GaN/cubic SiC/ $\mathrm{Si}$ heterostructures for vertical devices," Journal of Crystal Growth, vol. 311-10, Article ID 2840, 2009.

[37] P. V. Seredin, D. L. Goloshchapov, D. S. Zolotukhin et al., "Electronic and optical properties of hybrid GaN/por-Si (111) heterostructures," Quantum Electron, vol. 49, Article ID 545, 2019.

[38] P. T. Huong, M. Idrees, B. Amin et al., "Electronic structure, optoelectronic properties and enhanced photocatalytic response of $\mathrm{GaN}-\mathrm{GeC}$ van der Waals heterostructures: a first principles study," RSC Advances, vol. 10, Article ID 24127, 2020.

[39] S. H. Khan, R. Osla, R. Islam, S. M. Islam, and M. M. Ibrahim, "Tunable photocatalytic properties of planar GaN/GeC hetero-bilayer: production of $\mathrm{H}_{2}$ fuel," IEEE Access, vol. 8, Article ID 209030, 2020.

[40] P. Giannozzi, S. Baroni, N. Bonini et al., "Quantum ESPRESSO: a modular and open-source software project for quantum simulations of materials," Journal of Physics: Condensed Matter, vol. 21, p. 1, Article ID 395502, 2009.

[41] J. P. Perdew, K. Burke, and M. Ernzerhof, "Generalized gradient approximation made simple," Physical Review Letters, vol. 77, Article ID 3865, 1996.

[42] D. Vanderbilt, "Soft self-consistent pseudopotentials in a generalized eigenvalue formalism," Physical Review B, vol. 41, Article ID 7892, 1990.
[43] H. J. Monkhorst and J. D. Pack, "Special points for Brillouinzone integrations," Physical Review B, vol. 13, Article ID 5188, 1976.

[44] R. Ponce-Pérez, R. M. T. de la Cruz, S. J. Gutiérrez-Ojeda, J. Guerrero-Sánchez, J. Varalda, and G. H. Cocoletzi, "Initial stages of the epitaxial growth of $\mathrm{MnN}$ on the GaAs (001)$(2 \times 2)$ surface: first-principle study," Applied Surface Science, vol. 489, Article ID 639, 2019.

[45] J. C. Moreno, J. H. Camacho-García, R. Ponce-Pérez, F. Sánchez-Ochoa, M. T. R. de la Cruz, and G. H. Cocoletzi, "Initial stages of the epitaxial growth of AlN on GaN (111)$(2 \times 2)$ surface: ab-initio studies," Journal of Crystal Growth, vol. 507, Article ID 370, 2019.

[46] J. Guerrero-Sánchez, M. Lopez-Fuentes, F. Sánchez-Ochoa, N. Takeuchi, and G. H. Cocoletzi, "Nitrogen induced phosphorene formation on the boron phosphide (111) surface: a density functional theory study," RSC Advances, vol. 6-110, Article ID 108621, 2016.

[47] J. Guerrero-Sanchez, F. Sanchez-Ochoa, G. H. Cocoletzi, J. F. Rivas-Silva, and N. Takeuchi, "Ab-initio studies of the Sc adsorption and the $\mathrm{ScN}$ thin film formation on the $\mathrm{GaN}(000$ 1)-(2×2) surface," Thin Solid Films, vol. 548, Article ID 317, 2013.

[48] M. T. Romero, G. H. Cocoletzi, and N. Takeuchi, "First principles calculations of the Sc adsorption on Si (001)-c $(2 \times 4)$," Surface Science, vol. 606, Article ID 1382, 2010.

[49] W. Z. Yu, J. A. Yan, and S. P. Gao, "Band gap characters and ferromagnetic/antiferromagnetic coupling in group-IV monolayers tuned by chemical species and hydrogen adsorption configurations," Nanoscale Research Letters, vol. 10, p. 1, Article ID 351, 2015.

[50] Z. Xu, Y. Li, C. Li, and Z. Liu, "First-principles molecular dynamics study on structural and electronic properties of amorphous germanium carbide," Journal of Non Crystal Solids.vol. 443, Article ID 125, 2016.

[51] G. X. Qian, R. M. Martin, and D. J. Chadi, "First-principles study of the atomic reconstructions and energies of Ga- and As-stabilized GaAs(100) surfaces," Physical Review B, vol. 38, no. 11, Article ID 7649, 1988.

[52] A. Mahmood, A. Shah, F. F. Castillon et al., "Surface analysis of $\mathrm{GeC}$ prepared by reactive pulsed laser deposition technique," Current Applied Physics, vol. 11, Article ID 547, 2011.

[53] J. Vilcarromero and F. C. Marques, "XPS study of the chemical bonding in hydrogenated amorphous germanium-carbon alloys," Applied Physics A, vol. 70, Article ID 581, 2000.

[54] C. Hu, W. Zheng, H. Tian, L. Xu, and Q. Jiang, "Effects of the chemical bonding on the optical and mechanical properties for germanium carbide films used as antireflection and protection coating of ZnS windows," Journal of Physics: Condensed Matter, vol. 18, Article ID 4231, 2006.

[55] L. Liu, Y. Diao, S. Xia, F. Lu, and J. Tian, "A first principle study on systematic stability and electronic properties of $\mathrm{GaN}$ nanowire surface with $\mathrm{Cs} / \mathrm{Li} / \mathrm{NF}_{3}$ co-adsorption," Applied Surface Science, vol. 478, Article ID 393, 2019. 